This item was submitted to Loughborough's Research Repository by the author.

Items in Figshare are protected by copyright, with all rights reserved, unless otherwise indicated.

\title{
Generation of flexural waves in plates by laser-initiated airborne shock
}

waves

PLEASE CITE THE PUBLISHED VERSION

http://dx.doi.org/10.1016/j.jsv.2010.08.005

PUBLISHER

(C) Elsevier

VERSION

AM (Accepted Manuscript)

LICENCE

CC BY-NC-ND 4.0

REPOSITORY RECORD

Georgiev, Vasil B., Victor V. Krylov, Qin Qin, and Keith Attenborough. 2012. "Generation of Flexural Waves in Plates by Laser-initiated Airborne Shock Waves”. figshare. https://hdl.handle.net/2134/10785. 
This item was submitted to Loughborough's Institutional Repository (https://dspace.lboro.ac.uk/) by the author and is made available under the following Creative Commons Licence conditions.

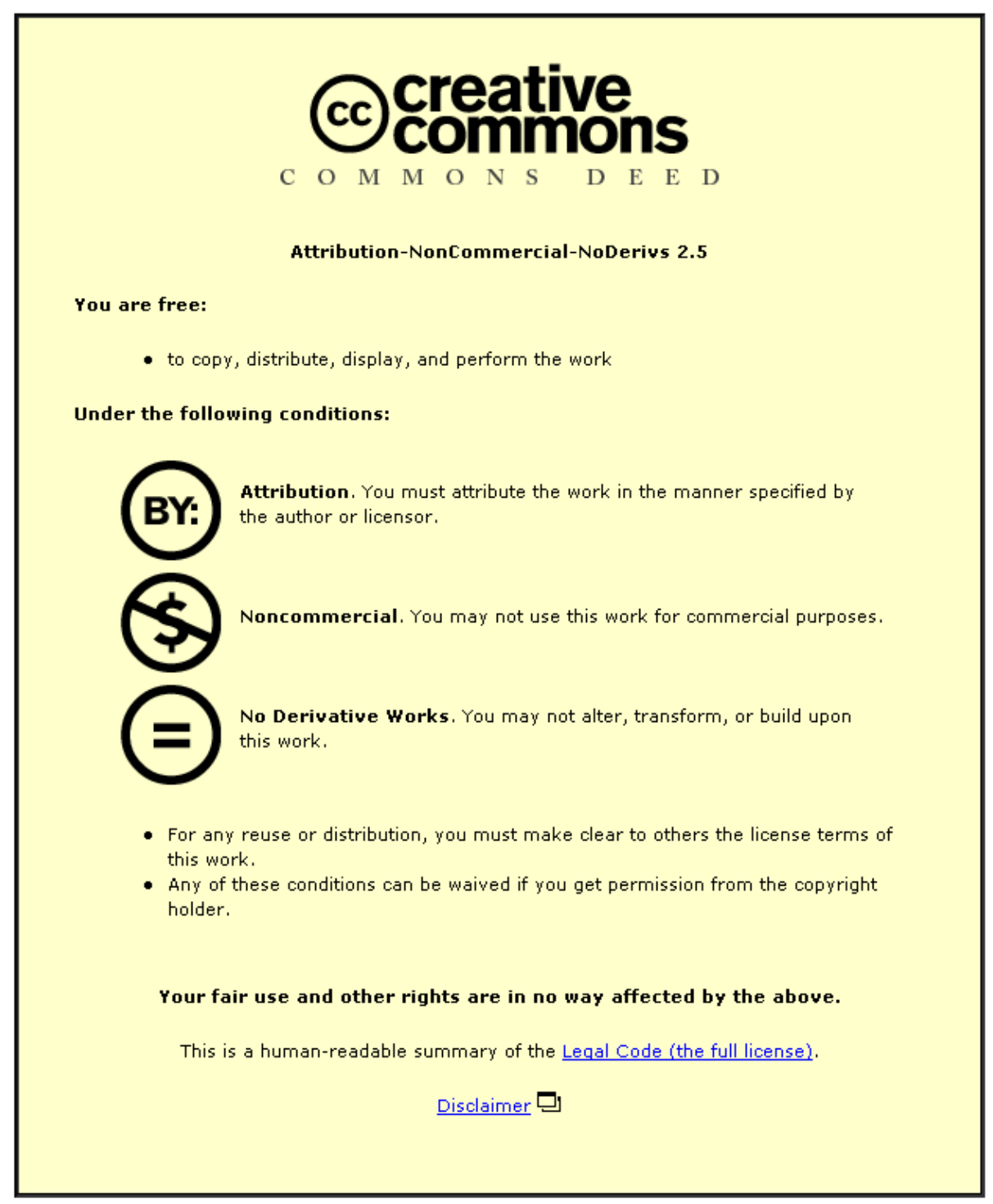

For the full text of this licence, please go to: http://creativecommons.org/licenses/by-nc-nd/2.5/ 


\section{Generation of flexural waves in plates by laser-}

\section{initiated airborne shock waves}

Vasil B. Georgiev ${ }^{\text {a }}$, Victor V. Krylov ${ }^{\text {a,* }}$, Qin Qin ${ }^{\text {, }}$, Keith Attenborough ${ }^{\text {c }}$,

${ }^{\mathrm{a}}$ Department of Aeronautical and Automotive Engineering, Loughborough University, Ashby Road, Loughborough, Leicestershire, LE11 3TU, UK;

${ }^{\mathrm{b}}$ Acoustic Research Centre, Department of Engineering, University of Hull, Cottingham Road, Hull, HU6 7RX, UK;

${ }^{\mathrm{C}}$ Department of Engineering, University of Hull, Cottingham Road, Hull, HU6 7RX, UK.

\section{Abstract}

Results of the semi-analytical modelling of the interaction of laser-initiated airborne shock waves with an infinite horizontally positioned elastic plate are presented. The impact of the airborne shock waves on the plate is approximated by a cylindrically diverging surface force resulting from the pressure of the incident and reflected shock waves. This force is then represented in the wavenumber-frequency domain by means of Hankel and Fourier transforms that are carried out numerically so that the interaction problem can be solved using the Green's function method. The resulting frequency spectra and time histories of generated 
flexural wave pulses are calculated for different values of laser pulse energy and for different heights of the laser beam focusing above the plate surface. The theoretical results obtained are compared with the results of laboratory measurements of the interaction of laser-generated acoustic shocks with a large plastic plate. The comparison shows reasonably good agreement between the semi-analytical predictions and data.

Keywords: Flexural waves in plates, wave generation, laser-initiated airborne shock waves, semi-analytical approach.

\section{Introduction}

Controlled explosions that take place above ground surface during military testing generate not only shock waves in air but also strong ground vibrations [1, 2]. Experimental studies of the propagation of such shock waves and shock-induced ground vibrations are relatively scarce because field trials with explosions are expensive and difficult. It is convenient and much less expensive to study the associated sound and vibration phenomena using the reduced-scale laboratory simulations using sparks produced by a focused high energy pulsed laser as sources of airborne shock waves interacting with large elastic plates modelling the ground $[3,4]$.

Although there have been several investigations of laser-generated elastic waves in plates in the past [5-7], in all these investigations a focused laser beam interacted directly with a plate, and flexural and quasi-longitudinal plate waves were generated by means of the so-

\footnotetext{
* Corresponding author. Tel.: +44 1509 227216; E-mail address: V.V.Krylov@lboro.ac.uk
} 
called thermo-optical generation mechanism associated with the conversion of light energy into heat and subsequent thermal expansion of the heated area. Due to a small size of the heated area of the resulting thermo-optical sources, the physical nature of this situation is very similar to wave generation in plates by mechanical concentrated forces [8-10]. However, this picture is not adequate for experiments $[3,4]$, where a laser beam is focused in the air outside a plate, since laser-initiated air-shock wave interaction with a plate involves a rather complex and essentially distributed source.

The use of a strong shock wave in air as a distributed source for elastic wave generation in solids has been investigated in respect of generation of Rayleigh surface waves by electric spark discharge near the surface [11]. In that investigation, a semi-analytical model describing interaction of air shock waves with an elastic half space was developed using an exponential function to approximate the air pressure in the shock wave.

The aim of the present paper is to develop the above-mentioned semi-analytical model [11] further and to apply it to the interaction of laser-initiated airborne shock waves with elastic plates. Some of the results of this paper have been presented at the UK Institute of Acoustics Spring Conference 2008 in Reading [12] and at the conference 'Acoustics -08' in Paris [13].

The impact of the incident shock wave is approximated by an equivalent cylindrically diverging surface force resulting from the surface pressure of the incident and reflected shock waves. The effect of non-linear reflection of a shock wave is included into the theoretical model as well. The well-known analytical expressions for air particle velocity and pressure in the front of a shock wave are used to describe this surface force as a function of time and distance from the epicentre. The shock wave pressure behind the front is approximated as a combination of two exponential functions in order to model positive and negative phases of a shock wave typical for larger distances between the shock wave origin and the point of 
observation (see also [12, 13]). The problem is then solved using the Green's function method applied to an infinite plate. The resulting frequency spectra and time histories of the generated flexural wave pulses are calculated for different values of the height of the laser-generated spark above the plate surface. The theoretical results for time histories and frequency spectra of generated flexural waves are compared with the results of the reduced-scale model experiments on laser-initiated air shock wave interaction with a large plastic plate.

\section{Theoretical background}

\subsection{Specification of the equivalent surface force}

According to [11], the pressure in the strong spherically diverging airborne shock wave can be approximated by the following analytical expression:

$$
P^{s h}(r, t)=P^{s h}(r) H\left(t-\int_{0 v^{s h}}^{r} \frac{d r^{\prime}}{\left.s r^{\prime}\right)}\right) \exp \left[-\left(t-\int_{0 v^{s h}\left(r^{\prime}\right)}^{r} \frac{d r^{\prime}}{\varepsilon \int_{0} \frac{r}{s h}\left(r^{\prime}\right)}\right)^{-1}\right],
$$

Here $H(t)$ is a Heavyside step function, $v^{\text {sh }}(r)$ and $P^{s h}(r)$ are wave velocity and pressure in the front of a shock wave [14] and $\varepsilon=0.5$ is a constant controlling the duration of the pressure pulse:

$$
v^{s h}(r)=\frac{2}{5} \zeta_{0}^{5 / 2}\left(\frac{E}{\rho_{0}}\right)^{1 / 2} r^{-3 / 2}
$$




$$
P^{s h}(r)=\left(\frac{2}{5}\right)^{2} \frac{2 \zeta_{0}^{5}}{\gamma+1} E r^{-3}
$$

$E$ is the energy instantly released in the origin of the shock wave, $\rho_{0}$ is the mass density of the air, $\gamma \approx 1.41$ is the ratio of specific heats, and $\zeta_{0} \approx 0.93$ is a dimensionless parameter characterizing the self-similar motion of the wave front [14].

As a result of interaction of the shock wave with an infinite plate, a considerable part of the incident wave energy is reflected back into the air, whereas the remainder is transformed into the energy of flexural plate vibrations. To evaluate the air pressure acting on the plate surface, the latter can be approximated as absolutely rigid. In this case, the surface pressure can be considered as the sum of the incident and reflected pressures. Using the usual linear acoustic reflection coefficient from a rigid surface (equal to 1), the resulting surface pressure can be expressed simply as twice the pressure in the incident wave. Thus, the equivalent normal surface force per unit area $F_{n}^{\text {sh }}$ created by the shock wave can be expressed, using Eqns (1) - (3), as

$$
F_{n}^{s h}(t, \rho)=2 P^{s h}(r) H\left[t-t_{0}^{s h}\right] \exp \left\{-\left[t-t_{0}^{s h}\right] / \varepsilon t_{0}^{s h}\right\}
$$

where

$$
t_{0}^{s h}=\left(\frac{r}{\zeta_{0}}\right)^{5 / 2}\left(\frac{\rho_{0}}{E}\right)^{1 / 2}
$$


In Eqn (4), the distance $r$ from the origin of laser-induced acoustic shock to a point of observation on the surface is equal to $\sqrt{\rho^{2}+h^{2}}$ (see Fig. 1).

For further analysis, the frequency spectrum of the normal force $F_{n}^{\text {sh }}(t, \rho)$ is required:

$$
F_{n}^{s h}(\omega, \rho)=\frac{1}{2 \pi} \int_{-\infty}^{+\infty} F_{n}^{s h}(t, \rho) \exp (i \omega t) d t
$$

Substituting Eqn (4) into Eqn (5), this frequency spectrum can be expressed as follows:

$$
F_{n}^{s h}(\omega, \rho)=\frac{P^{s h}(r)}{\pi} \frac{\varepsilon t_{0}^{s h}}{1-i \omega \varepsilon t_{0}^{s h}} \exp \left(i \omega t_{0}^{s h}\right)
$$

\subsection{Modified equivalent surface force}

In the previous section, the equivalent surface force (Eqn (4)) has been specified using Eqns (1)-(3). The pressure due to the air shock wave in this description is an exponential function with its maximum value depending on the distance to the center of the laser-induced spark. The time history of the air pressure pulse calculated according to Eqns (1)-(3) can be seen in Fig. 2 (a). Note however that for larger distances between the shock wave origin and a surface, which are of primary interest in this paper, both analytical modeling and measurements show that pressure pulses induced by shock waves behave in a more complex way. In particular, when the shock front approaches a given point, the pressure initially undergoes a discontinuous jump above atmospheric pressure before decreasing below 
atmospheric pressure (positive and negative phases), and finally returning to its initial value [14].

Consequently, Eqn (1) has been modified to model shock wave pulses for larger distances by adding an additional exponential term, that helps to describe the negative pressure phase The air pressure can then be expressed as follows (see also Fig. 2 (b)):

$$
P^{s h}(r, t)=P^{s h}(r)\left\{\begin{array}{l}
H\left(t-t_{0}^{s h}\right)\left[1.2 \exp \left(-\left(t-t_{0}^{s h}\right) \frac{1}{\varepsilon t_{0}^{s h}}\right)-0.2\right]- \\
H\left(t-t_{0}^{s h} 3.5\right)\left[1.2 \exp \left(-\left(t-t_{0}^{s h}\right) \frac{0.36}{\varepsilon t_{0}^{s h}}\right)-0.2\right]
\end{array}\right\} .
$$

Using the above-mentioned modified expression for air pressure (Eqn. (7)) and the acoustic approximation for the reflection coefficient, the equivalent surface force is given by:

$$
F_{n}^{s h}(r, t)=2 P^{s h}(r, t),
$$

Following Fourier transform, the frequency spectrum of the above function can be expressed as follows:

$$
F_{n}^{s h}(\omega, \rho)=\frac{P^{s h}(r)}{\pi}\left\{\begin{array}{l}
\frac{1.2 \varepsilon t_{0}^{s h}}{1-i \omega \varepsilon t_{0}^{s h}} \exp \left(i \omega t_{0}^{s h}\right)-\frac{0.2}{i \omega}\left[\exp \left(i \omega t_{0}^{s h} 3.5\right)-\exp \left(i \omega t_{0}^{s h}\right)\right] \\
-\frac{1.2 \varepsilon t_{0}^{s h}}{0.36-i \omega \varepsilon t_{0}^{s h}} \exp \left(-\frac{0.9}{\varepsilon}+i \omega t_{0}^{s h} 3.5\right)
\end{array}\right\} .
$$


Note that the above expressions for the equivalent surface force (Eqns (6) and (8)) have been derived using Eqns (2) and (3) for the velocity and pressure of a strong air shock wave [8]. However, for distances, $r$, that are large enough, say $r>r_{0}$, where $\mathrm{r}_{0}$ is the distance at which the velocity of a shock front becomes equal to the speed of sound $v_{0}$, the abovementioned expressions for surface forces are no longer valid. Figure 3 (a) shows how the velocity of the shock front changes with the distance from the origin. In this Figure, the velocity of a shock wave front has been plotted as a function of horizontal distance from the epicentre $\rho$ according to Eqn (2) in which $r=\sqrt{\rho^{2}+h^{2}}$ (height $h$ was considered as a parameter).

In this case, beyond the distance $r_{0}=0.0084 \mathrm{~m}$ (defined from Eqn (2) by assuming $\mathrm{v}^{\mathrm{sh}}(\mathrm{r}$ ) $=v_{0}=340 \mathrm{~m} / \mathrm{s}$ and $E=0.8 \mathrm{~J}$ (the laser energy per pulse)), the air particle velocity and pressure have to be calculated using the characteristics of a normal acoustic wave propagating with the speed of sound, $v_{0}$. As is well known, the wave pressure in this case is inversely proportional to the distance: $P(r)=A / r$, where the constant $A$ should be determined by equalizing the air pressure in the shock wave to the acoustic wave pressure at $r=r_{0}$. Moreover, the time duration of a pressure pulse for a strong shock wave increases proportionally to the distance from the origin, whereas in a normal acoustic wave this duration is constant. Thus the time duration of the pressure pulses at distance $r=r_{0}$ should be equalized as well. For an acoustic wave, Eqns (2) and (3) should be replaced by the following expressions:

$$
\begin{gathered}
v^{a c}=v_{0}=340 \mathrm{~m} / \mathrm{s} \\
P^{a c}(r)=\left(\frac{2}{5}\right)^{2} \frac{2 \zeta_{0}^{5}}{\gamma+1} \frac{E}{r_{0}^{2} r}
\end{gathered}
$$


Using the above equations, one can obtain the following expression for $r>r_{0}$ :

$$
P^{a c}(r, t)=P^{a c}(r)\left\{\begin{array}{l}
H\left(t-t_{0}^{a c}\right)\left[1.2 \exp \left(-\left(t-t_{0}^{a c}\right) \frac{1}{\varepsilon t_{0}^{s h}\left(r_{0}\right)}\right)-0.2\right]- \\
H\left(t-t_{0}^{a c}-2.5 t_{0}^{s h}\left(r_{0}\right)\right)\left[1.2 \exp \left(-\left(t-t_{0}^{a c}\right) \frac{0.36}{\varepsilon t_{0}^{s h}\left(r_{0}\right)}\right)-0.2\right]
\end{array}\right\},
$$

where $t_{0}^{a c}=t_{0}^{s h}\left(r_{0}\right)+\frac{r-r_{0}}{v_{0}}$.

Figure 3 (b) shows the pressure pulse calculated according to Eqn (10) at distances of 2 and $4 \mathrm{~cm}$ from the laser-initiated spark. For comparison, Fig. 4 (a) shows the corresponding experimental pressure pulse generated by a Q-switch Surelite III-10 Nd: YAG laser with the same energy of $0.8 \mathrm{~J}$ per pulse [4] and at the distance of $3 \mathrm{~cm}$ from the spark (see the experimental section of this paper for more details of the laser source). It can be seen that while the predicted and measured waveforms agree with each other quite well, both in shape and duration of positive parts, the measured peak amplitude is about two times smaller than the predicted one. However, given the approximations used in the process of modelling, especially in the transition zone from a strong shock wave to an acoustic wave, and the neglect of material losses during wave propagation, this agreement is considered reasonable.

Using again the linear acoustics approximation for the reflection coefficient, the equivalent normal surface force can assumed, initially, to be the doubled incident pressure, and the frequency spectrum of the equivalent surface force can be expressed as: 


$$
F_{n}^{a c}(\omega, \rho)=\frac{P^{a c}(r)}{\pi}\left\{\begin{array}{l}
\frac{1.2 \varepsilon t_{0}^{s h}\left(r_{0}\right)}{1-i \omega \varepsilon t_{0}^{s h}\left(r_{0}\right)} \exp \left(i \omega t_{0}^{a c}\right)-\frac{0.2}{i \omega}\left[\exp \left(i \omega\left(t_{0}^{a c}+t_{0}^{s h}\left(r_{0}\right)\right)\right)-\exp \left(i \omega t_{0}^{a c}\right)\right] \\
-\frac{1.2 \varepsilon t_{0}^{s h}\left(r_{0}\right)}{0.36-i \omega \varepsilon t_{0}^{s h}\left(r_{0}\right)} \exp \left(-\frac{0.9}{\varepsilon}+i \omega\left(t_{0}^{a c}+2.5 t_{0}^{s h}\left(r_{0}\right)\right)\right)
\end{array}\right\}
$$

\subsection{Accounting for non-linear reflection}

So far the equivalent surface force has been expressed using a linear coefficient of reflection equal to 1 , which means that the pressure on the infinite plate is assumed to be twice the pressure in the incident shock wave. For shock waves, the problem of reflection is non-linear, and the reflection coefficient depends on the amplitude of the shock wave. Figure 5 shows the reflection coefficient as function of the incident overpressure, $p_{1} / p_{0}$, where $p_{1}$ is the pressure in the shock wave, whereas $p_{0}$ is the atmospheric pressure. The graphs correspond to air, $\gamma=1.41$, and to monatomic gas, $\gamma=5 / 3$.

The graphs in Fig. 5 have been calculated using a simple formula given by Wright [15]:

$$
\frac{\text { reflected_overpressure }}{\text { incident_overpressure }}=\frac{p_{2}-p_{0}}{p_{1}-p_{0}}=\frac{(2 \mu+1) y+1}{\mu y+1},
$$

where $y=p_{1} / p_{0}$ and $\mu=(y-1) /(y+1)$.

In the experimental work presented in Section 3, the maximum strength of the incident overpressure has been achieved for the height of the spark $0.003 \mathrm{~m}$ and laser energy $0.8 \mathrm{~J}$ per 
pulse, and it can be evaluated to be about 27. Thus, for further consideration, the reflection coefficient could be approximated using a simple linear function, such as: $R=a+b y$. The coefficients $a$ and $b$ can be determined from Fig. 5 ( $a=1, b=0.21)$. In this case the resulting surface pressure can be expressed in the form:

$$
P_{\text {surf }}=P_{\text {in }}+R P_{\text {in }}=(1+a) P_{\text {in }}+b \frac{P_{\text {in }}^{2}}{P_{a t m}}
$$

For the sake of simplicity, the effect of non-linear reflection will be studied here using the simpler pressure expression, Eq. (1) (see [11]). In this case the equivalent surface force taking the nonlinear reflection into account can be written as:

$$
F_{n}^{s h}(t, r)=(1+a) P^{s h}(r) H\left(t-t_{0}^{s h}\right) \exp \left(-\frac{t-t_{0}^{s h}}{\varepsilon t_{0}{ }^{s h}}\right)+\frac{b}{P_{a t m}}\left[P^{s h}(r)\right]^{2} H\left(t-t_{0}^{s h}\right) \exp \left[-\frac{2\left(t-t_{0}^{s h}\right)}{\varepsilon t_{0}^{s h}}\right]
$$

whereas its frequency spectrum is given as follows:

$$
F_{n}^{s h}(\omega, r)=\frac{(1+a) P^{s h}(r)}{2 \pi} \frac{\varepsilon t_{0}^{s h}}{1-i \omega \varepsilon t_{0}^{s h}} \exp \left(i \omega t_{0}^{s h}\right)+\frac{b\left[P^{s h}(r)\right]^{2}}{2 \pi P_{a t m}} \frac{\varepsilon t_{0}^{s h}}{2-i \omega \varepsilon t_{0}^{s h}} \exp \left(i \omega t_{0}^{s h}\right)
$$

\subsection{Flexural wave generation in an infinite plate}

Since the problem under consideration is axi-symmetric, the flexural response of the infinite plate to the spectral component of the applied normal force $F_{n}(x, y, \omega)$ due to the 
airborne shock wave can be described by the plate equation in cylindrical coordinates, with the origin at the epicentre [16]:

$$
D\left(\frac{d^{2}}{d \rho^{2}}+\frac{1}{\rho} \frac{d}{d \rho}\right)^{2} w-\rho_{S} d \omega^{2} w=F_{n}(\omega, \rho)
$$

where

$$
F_{n}(\omega, \rho)=\left\{\begin{array}{ll}
F_{n}^{s h}, & 0<r<r_{0} \\
F_{n}^{a c}, & r>r_{0}
\end{array}\right\}
$$

Here $D=\widetilde{E} d^{3} / 12\left(1-v^{2}\right)$ is bending stiffness of the plate, $\widetilde{E}$ is the Young's modulus, $d$ and $\rho_{s}$ are the plate thickness and mass density respectively, and $v$ is the Poisson's ratio. Equations (16) and (17) can be solved using Green's function method, which yields the following expression for bending displacement in the wavenumber-frequency domain [16]:

$$
\tilde{w}(\omega, k)=\frac{F_{n}(\omega, k)}{D\left(k^{4}-k_{f}^{4}\right)} .
$$

Here $k_{f}=\left(\rho_{s} d \omega^{2} / D\right)^{1 / 4}$ is the plate flexural wavenumber, and $F_{n}(\omega, k)$ is the Hankel transform of Eqn (17), which is to be calculated numerically:

$$
F_{n}(\omega, k)=\int_{0}^{\rho_{0}} F_{n}^{s h}(\omega, \rho) \rho J_{0}(k \rho) d \rho+\int_{\rho_{0}}^{\infty} F_{n}^{a c}(\omega, \rho) \rho J_{0}(k \rho) d \rho
$$


The inverse Hankel transform describes the plate response to the acoustic shock in the space-frequency domain:

$$
w(\omega, \rho)=\frac{1}{D} \int_{0}^{\infty} \frac{F_{n}(\omega, k) k J_{0}(k \rho) d k}{k^{4}-k_{f}^{4}} .
$$

Expressing the Bessel function in terms of Hankel functions and using the properties of Hankel functions, the bending displacement can be rewritten as:

$$
w(\omega, \rho)=\frac{1}{2 D} \int_{-\infty}^{\infty} \frac{F_{n}(\omega, k) k H_{0}^{(1)}(k \rho) d k}{k^{4}-k_{f}^{4}}
$$

The integral in Eqn (21) can be evaluated using the method of residues:

$$
w(\omega, \rho)=2 \pi i\left(\frac{1}{2 D} \frac{F_{n}\left(\omega, k_{f}\right) k_{f} H_{0}^{(1)}\left(k_{f} \rho\right)}{4 k_{f}^{3}}\right),
$$

where the term in brackets represents the residue at $k=k_{f}$. Using the usual asymptotic formula for the Hankel function at $k_{f} \rho>>1$, the expression for plate vertical displacement can be written as follows:

$$
w(\omega, \rho)=\frac{i F_{n}\left(\omega, k_{f}\right)}{4 \omega^{5 / 4} \rho_{S}^{5 / 8} d^{5 / 8} D^{3 / 8}} \sqrt{\frac{2 \pi}{\rho}} \exp \left(i k_{f} \rho-i \pi / 4\right) .
$$


This expression, combined with the harmonic time factor $e^{-i \omega t}$, represents a cylindrical flexural wave propagating from the epicenter of the acoustic shock. Obviously, the vertical components of plate particle velocity and acceleration can be obtained from Eqn. (23) as:

$$
\dot{w}(\omega, \rho)=-i \omega w(\omega, \rho) \text { and } \ddot{w}(\omega, \rho)=-\omega^{2} w(\omega, \rho)
$$

The time history of the displacement can be calculated from Eqn (23) using the inverse Fourier transform:

$$
w(t, \rho)=\int_{-\infty}^{\infty} w(\omega, \rho) e^{-i \omega t} d \omega
$$

Note that in our choice of the elementary Kirchhoff theory of plate vibrations (see Eqn (16) and the resulting Eqns (20-(23)) we followed the widely used criterion of its acceptability: $\left(d / \lambda_{t}\right)<0.05$, where $\lambda_{t}$ is the shear wavelength and $d$ is the plate thickness (e.g. [16]). For the parameters of the experimental plate (see Section 3 for more detail) and for a typical frequency of $10 \mathrm{kHz}$, the value of $\lambda_{t}$ at this frequency is $14.6 \mathrm{~cm}$. For the plate of 5 mm thickness, this gives $\left(d / \lambda_{t}\right)=0.034$, which satisfies the above criterion. For frequencies higher than $15 \mathrm{kHz}$ though, Eqns (16) and (20)- (23) may result in noticeable errors. Therefore, the use of the more precise Timoshenko-Mindlin plate theory would be recommended at such higher frequencies.

\section{Experimental results and their comparison with predictions}


The rig used for the model experiments is shown in Fig. 1 (b). Sparks were generated by focusing a beam from a Q-switch Surelite III-10 Nd: YAG laser with a $1064 \mathrm{~nm}$ wavelength and with the energy of $0.8 \mathrm{~J}$ per pulse $[3,4]$. The gas breakdown induced by the laser had a duration of between 4 and 6 nanoseconds. For this duration of the pulse the pulse power is between 133 and 200 MW. A convex lens with focal length of $10 \mathrm{~cm}$ has been used to focus the beam to a spot of diameter of about $0.3 \mathrm{~mm}$ so that the light intensity in the focused spot is between $1.88 \times 10^{11}$ and $2.83 \times 10^{11} \mathrm{~W} / \mathrm{cm}^{2}$. At the distance of $3 \mathrm{~cm}$ from the spark, the peak pressure of the laser-generated airborne shock wave measured by a microphone was $181 \mathrm{~dB}$ re $20 \mu \mathrm{Pa}$ (22683 Pa), as can be seen in Fig. 4 (a). As was mentioned above, for these values of laser pulse power and distance, the shock wave becomes in fact the acoustic wave. The frequency spectrum of this wave lies between $3 \mathrm{kHz}$ and $159 \mathrm{kHz}$, with a peak at $20 \mathrm{kHz}$ (see Fig. 4 (b)).

The analysis system used in the experiments consisted of a conditioning amplifier, B\&K Type 2636; an NI 5911 data acquisition card enabling sampling rates as high as 100 million samples per second and LabView software.

For the measurements of flexural vibrations, a DJB A/20 accelerometer with the mass $18 \mathrm{~g}$, sensitivity $35 \mathrm{pC} / \mathrm{g}$ and resonant frequency of $28 \mathrm{kHz}$ was used. The plate was made of laminated plastic material. Its horizontal dimensions and thickness were 1 x $1 \mathrm{~m}^{2}$ and $0.005 \mathrm{~m}$ respectively. The support for the plate was provided by a porous foam layer. Strictly speaking, the boundary conditions on the bottom side of the plate supported by the foam are not stress free. However, the effect of the foam is rather small and can be neglected. Further details about the laboratory equipment and test rig can be found elsewhere [3,4].

Some of the results of the theoretical modelling and experimental simulations are presented and compared in Figs. 6 and 7. The material characteristics used in the calculations 
are as follows: mass density $\rho_{s}=900 \mathrm{~kg} / \mathrm{m}^{3}$, Young's modulus $\tilde{E}=50 \cdot 10^{8} \mathrm{~N} / \mathrm{m}^{2}$, Poisson's ratio $v=0.3$ and speed of sound in air $v_{0}=340 \mathrm{~m} / \mathrm{s}$.

The results shown in Figs. 6 and 7 represent the time histories and amplitude spectra of the plate acceleration at distances $0.3 \mathrm{~m}$ and $0.6 \mathrm{~m}$ away from the epicenter. The heights of the laser-generated spark above the plate surface, $\mathrm{h}$, were $0.003 \mathrm{~m}$ and $0.039 \mathrm{~m}$.

It can be seen from Figs. 6 and 7 that the theoretical results agree well with the experimental ones for $\mathrm{h}=0.039 \mathrm{~m}$ at both values of the distance from the epicenter. Note that in the theoretical time histories the time delay is shown due to the air shock wave propagation from the spark area to the plate surface. In the measured time histories this time delay is not shown. Both theoretical and experimental time histories and frequency spectra reveal oscillations at frequency about $5 \mathrm{kHz}$. Apparently, this behavior is due to the well-known coincidence phenomenon. The latter can occur when sound waves incident on a plate from the air at a particular angle have a trace wavelength matching exactly to that of the plate bending waves. The coincidence frequency is determined by the well-known formula (see e.g. [16]):

$$
f_{c}=\frac{c^{2}}{2 \pi} \sqrt{\frac{12 \rho_{s}\left(1-v^{2}\right)}{\tilde{E} d^{2}}}
$$

According to the above expression, the coincidence frequency for the case under consideration is $5160 \mathrm{~Hz}$, which is in a very good agreement with the experimental and numerical results shown in Figs. 6 and 7. By changing thickness of the plate, one can easily demonstrate that the effect of the coincidence phenomenon on generated acceleration pulses could be eliminated. Figure 8 shows the numerically calculated acceleration responses and their spectral components for different values of plate thickness. For a plate thickness equal to $0.005 \mathrm{~m}$, as above, the coincidence-related oscillations are clearly seen (Fig. $8 \mathrm{a}$ ), and the 
coincidence frequency is about $5 \mathrm{kHz}$ (Fig. $8 \mathrm{~b}$ ), as mentioned above. For the values of thickness 0.05 and $0.5 \mathrm{~m}$, the spectral peak frequency gradually reduces to zero, in line with Eqn (25), and the oscillations disappear. In particular, Fig. 8 (e) shows a single pulse induced by the shock wave (the small oscillations after the pulse are due to the numerical calculations).

The agreement of the theory with the experiments for $h=0.003 \mathrm{~m}$ is not as good as for $\mathrm{h}$ $=0.039 \mathrm{~m}$. It is seen from Figs. 6 (c) and 7 (c) that, for $\mathrm{h}=0.003 \mathrm{~m}$, the measured frequency spectra, in addition to the above-mentioned peaks at the coincidence frequency, also have strong additional frequency peaks near $9 \mathrm{kHz}$ and $14 \mathrm{kHz}$. The nature of these additional peaks is yet unclear. As they are not present in the case of $\mathrm{h}=0.039 \mathrm{~m}$, it is likely that these additional peaks at $\mathrm{h}=0.003 \mathrm{~m}$ may be caused by nonlinear effects associated with the much larger amplitude of the shock wave at lower heights.

One of the possible causes for these additional peaks could be attributed to non-linear effects associated with reflection of shock waves. To check this assumption, the non-linear reflection of shock waves has been taken into account, according to Eqns (14) and (15). Figure 9 shows the comparison between acceleration responses calculated at distance $0.3 \mathrm{~m}$ from the epicenter due to the equivalent surface force without non-linear effects, Eqn (6), and with non-linear effects, Eq. (15).

It can be seen from Fig. 9 that, the non-linear reflection is predicted to only increase the amplitude and slightly change the shape of the pulse. This is because the non-linear reflection is important only in the zone of a strong shock wave. Beyond the critical distance $r_{0}$, the reflection coefficient is again linear and equal to 1 . As a result of this, the calculated time histories and amplitude spectra of generated plate flexural waves do not differ significantly from those calculated using linear reflection. 
Another possible cause of the above-mentioned peaks at 9 and $14 \mathrm{kHz}$ in the case of $\mathrm{h}=$ $0.003 \mathrm{~m}$ could be non-linear effects associated with propagation of plate waves of high amplitude generated by a shock wave in this case. This includes the effect of nonlinear distortion of the initially time-harmonic flexural waves during their propagation in the plate after their excitation by a laser-initiated shock wave. It is known that as a result of such nonlinear distortion caused by deviation of stress-strain relations in the plate material from the linear Hook's law the wave front is steepening with distance, which is associated with the emergence of wave harmonics, thus giving additional components in the observed frequency spectra. This assumption needs further investigation, including additional experiments for different values of $h$, which is beyond the scope of the present work.

\section{Conclusions}

In the present paper, a semi-analytical model of flexural wave generation in infinite plates by laser-initiated air shock waves has been developed. Analysis of the obtained results shows that the coincidence condition plays an important role in the observed phenomena, being responsible for harmonic oscillations of generated flexural wave signals around the coincidence frequency.

The comparison of the theoretical results with the laboratory measurements using lasergenerated sparks has shown reasonably good agreement, especially for the larger value of spark height over the plate surface $(\mathrm{h}=0.039 \mathrm{~m})$. This implies that the proposed semianalytical model is reliable enough for prediction of flexural wave generation in plates due to laser-initiated airborne shock waves. The observed discrepancy between the experimental and theoretical results for the case of $\mathrm{h}=3 \mathrm{~mm}$ - the presence of two additional frequency peaks 
in the experimental spectra, may be caused by non-linear effects for generated plate waves. These effects will suggest the need for a substantial amount of additional theoretical and experimental study.

\section{Acknowledgements}

The research reported here has been supported by EPSRC grant EP/E027121/1. 


\section{References}

[1] K. Attenborough, P. Schomer, F. v.d. Eerden, E. Vedy, Overview of the theoretical development and experimental validation of blast sound-absorbing surfaces, Noise Control Engineering Journal 53(3) (2006) 70 - 80.

[2] P. Schomer, K. Attenborough, Basic results from full-scale tests at Ft Drum, Noise Control Engineering Journal 53(3) (2006) 94 - 109.

[3] K. Attenborough, Q. Qin, Model experiments on shock wave interaction with the ground, Proceedings of the $19^{\text {th }}$ International Congress of Acoustics, Madrid, Spain, 2-7 September 2007 (on CD).

[4] Q. Qin, K. Attenborough, Characteristics and application of laser-generated acoustic shock waves in air, Applied Acoustics 65 (2004) 325-340.

[5] J. B. Spicer, A. D. W. McKie, J. W. Wagner, Quantitative theory for laser ultrasonic waves in a thin plate, Appl. Phys. Lett. 57(18) (1990) 1882-1884.

[6] J.-C. Cheng, Y. H. Berthelot, Theory of laser-generated transient Lamb waves in orthotropic plates, J. Phys. D: Appl. Phys. 29 (1996) 1857-1867. 
[7] H.S Hsieh, J. Lin, Laser-induced vibration during pulsed laser forming, Optics and Laser Technology 36(6) (2004) 431-439.

[8] N. Vasudevan, A. K. Mal, Response of an elastic plate to localized transient source, J. Appl. Mech. 52 (1985) 356-362.

[9] S. Schedin, C. Lambourg, A. Chaigne, Transient sound fields from impacted plates: comparison between numerical simulations and experiments, Journal of Sound and Vibration 221 (1999) 471-490.

[10] A.J. Berkhout, D. de Vries, M. C. Brink, Array technology for bending wave field analysis in constructions, Journal of Sound and Vibration 300 (2007) 25-42

[11] V.V. Krylov, On the theory of surface acoustic wave generation by electric spark discharge, J. Phys. D: Appl. Phys. 25 (1992) 155-161.

[12] V.B. Georgiev, V.V. Krylov, Q. Qin, K. Attenborough, Generation of flexural waves in infinite plates by laser-initiated air shock waves, Proceedings of the Institute of Acoustics 30, Pt. 2 (2008) 147-154.

[13] V.B. Georgiev, V.V. Krylov, Q. Qin, K. Attenborough, Semi-analytical modelling of plate flexural waves generated by laser initiated air shock waves, Proceedings of the International Conference “Acoustics 08”, Paris, France, June 2008, pp 1883-1888 (on CD). 
[14] Y.B. Zel'dovich, Y.P. Raizer, Physics of shock waves and high temperature hydrodynamic phenomena, Vols. 1 and 2, New York, Academic Press (1966, 1967).

[15] J.K. Wright, Shock tubes, New York, Wiley and Sons (1961).

[16] M.C. Junger, D. Feit, Sound, structures and their interaction, MIT Press, Cambridge, MA (1972). 


\section{Figure captions}

Fig. 1 Interaction of laser-initiated airborne shock waves with a plate: (a) - geometry of the problem, (b) - experimental scheme.

Fig. 2 Air pressure pulses calculated according to Eqn (1) - (a) and to Eqn (7) - (b).

Fig. 3 Shock front velocity as a function of height $h$ of the laser spark and distance $\rho$ from the epicenter - (a); pressure pulse in an acoustic wave according to Eqn (10) - (b).

Fig. 4 Measured laser-generated acoustic pulse (a) and the corresponding frequency spectrum (b) at a source-receiver distance of $3 \mathrm{~cm}$ [4]; the peak pressure is $181 \mathrm{~dB}$ (22683 Pa) and the positive duration is $12 \mu \mathrm{s}$ (reproduced from [4]).

Fig. 5 Reflection coefficient of a shock wave as function of the incident overpressure.

Fig. 6 Time histories ((a), (b)) and amplitude spectra ((c), (d)) of the plate acceleration at distance $0.3 \mathrm{~m}$ away from the epicenter: measured ((a), (c)) and calculated ((b),(d)).

Fig. 7 Time histories ((a), (b)) and amplitude spectra ((c), (d)) of the plate acceleration at distance 0.6 m away from the epicenter: measured ((a), (c)) and calculated ((b),(d)). 
Fig. 8 Time histories (right) and amplitude spectra (left) of the acceleration for different values of plate thickness

Fig. 9 Effects of linear (solid curves) and non-linear reflection (dashed curves) on time histories (a) and amplitude spectra (b) of plate acceleration. 


\section{Figures}

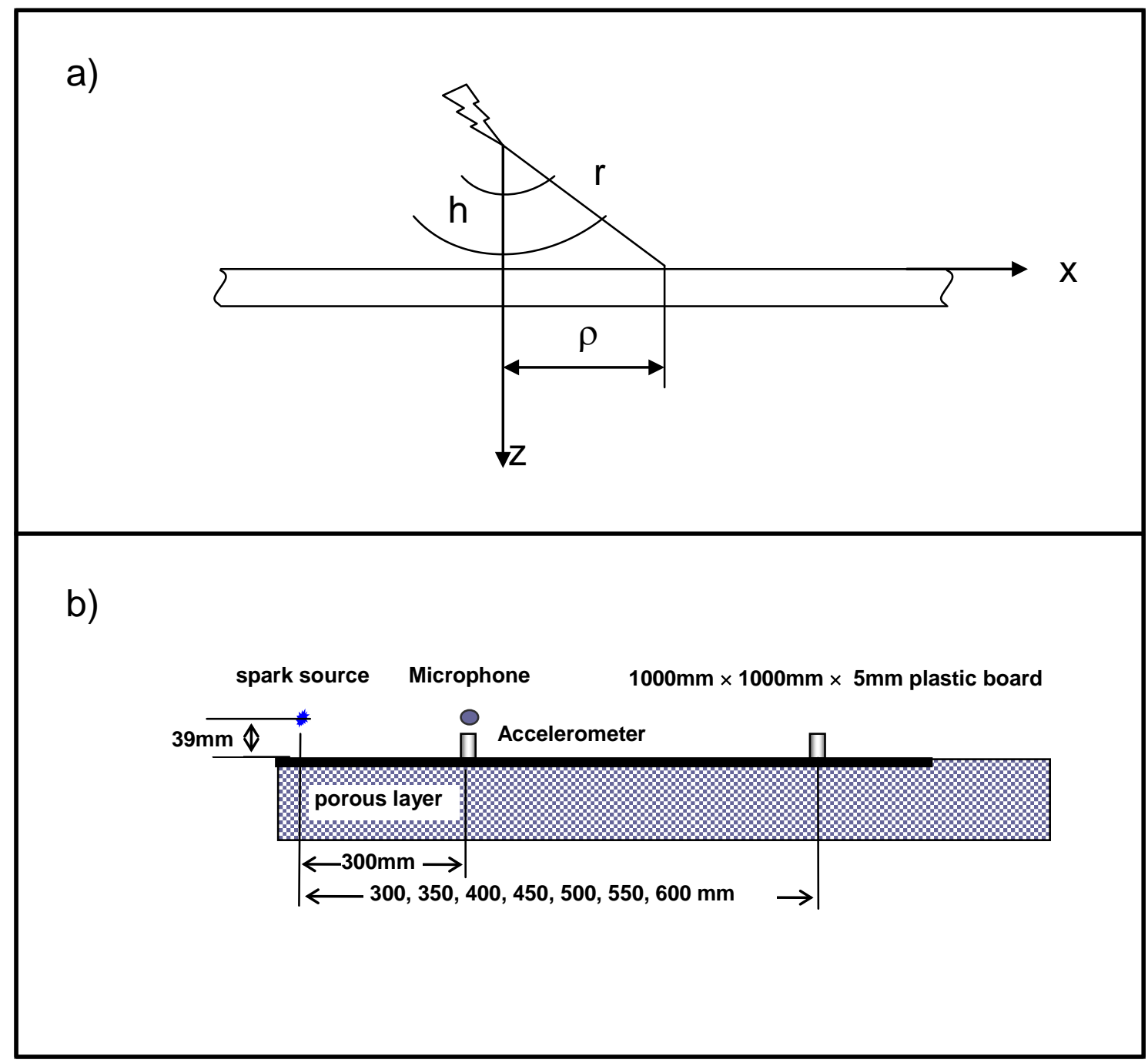

Fig. 1 Interaction of laser-initiated airborne shock waves with a plate: (a) geometry of the problem, (b) - experimental scheme. 


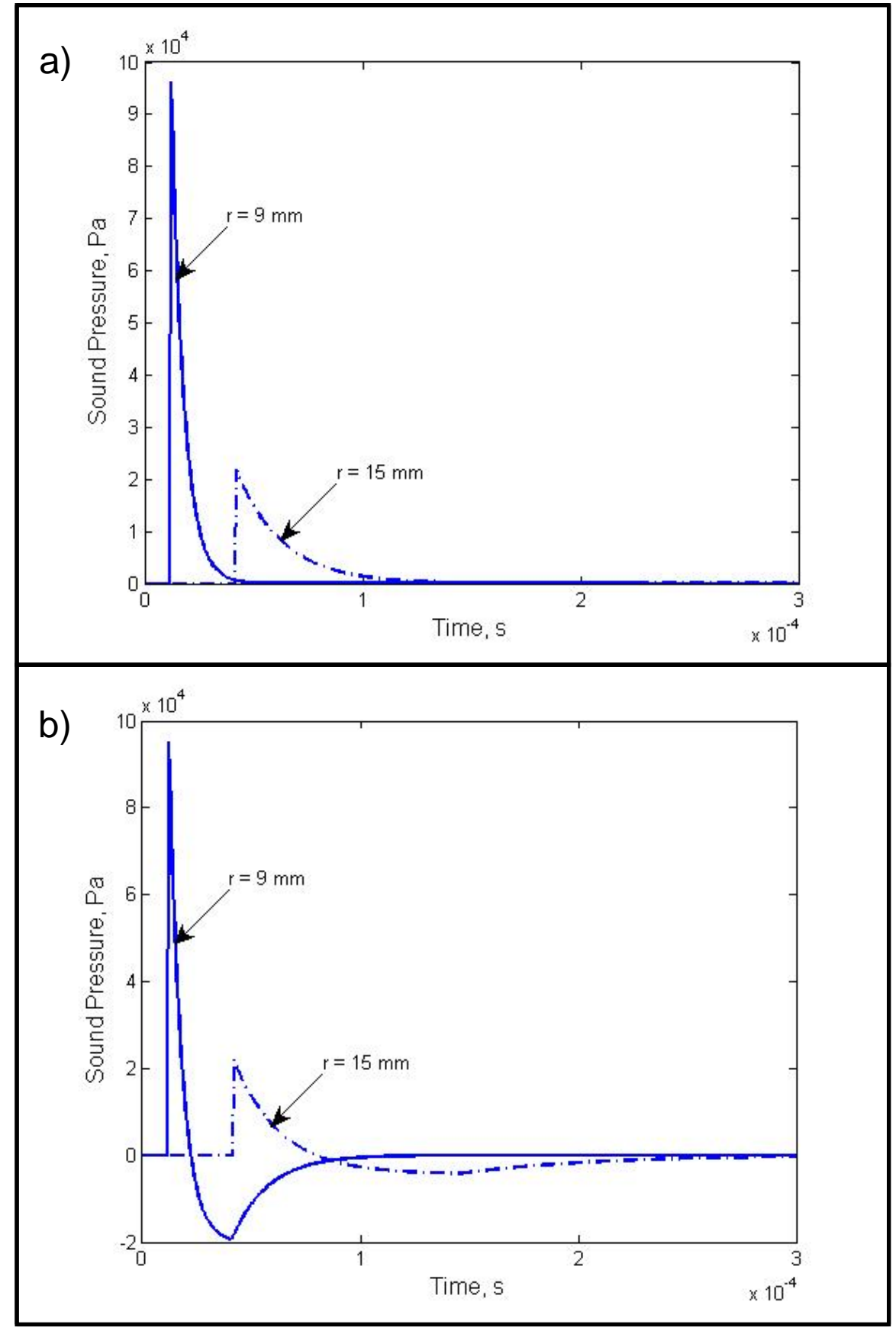

Fig. 2 Air pressure pulses calculated according to Eqn (1) - (a) and to Eqn (7) - (b). 


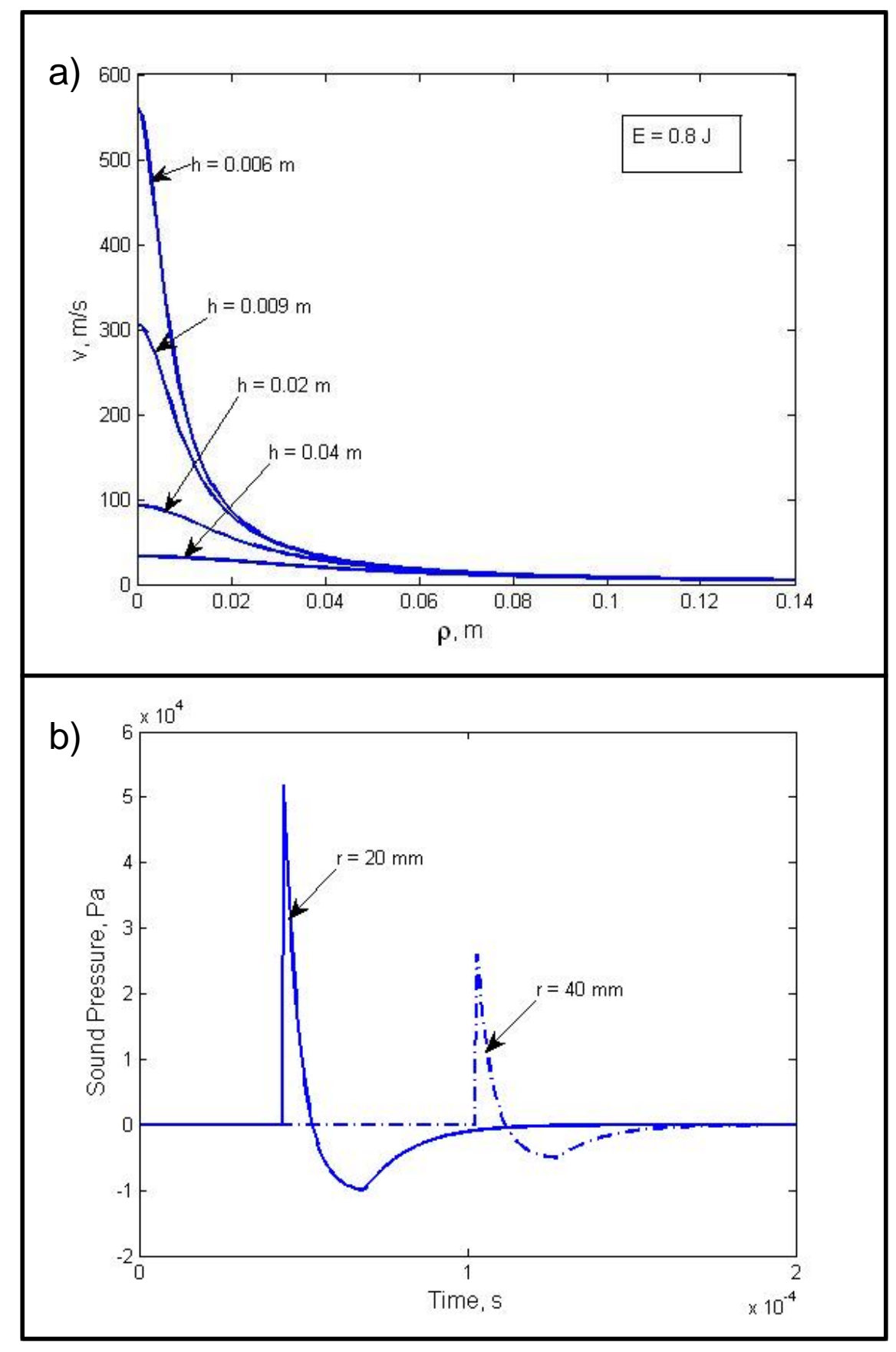

Fig. 3 Shock front velocity as a function of height $h$ of the laser spark and distance $\rho$ from the epicenter - (a); pressure pulse in an acoustic wave according to Eqn (10) - (b). 

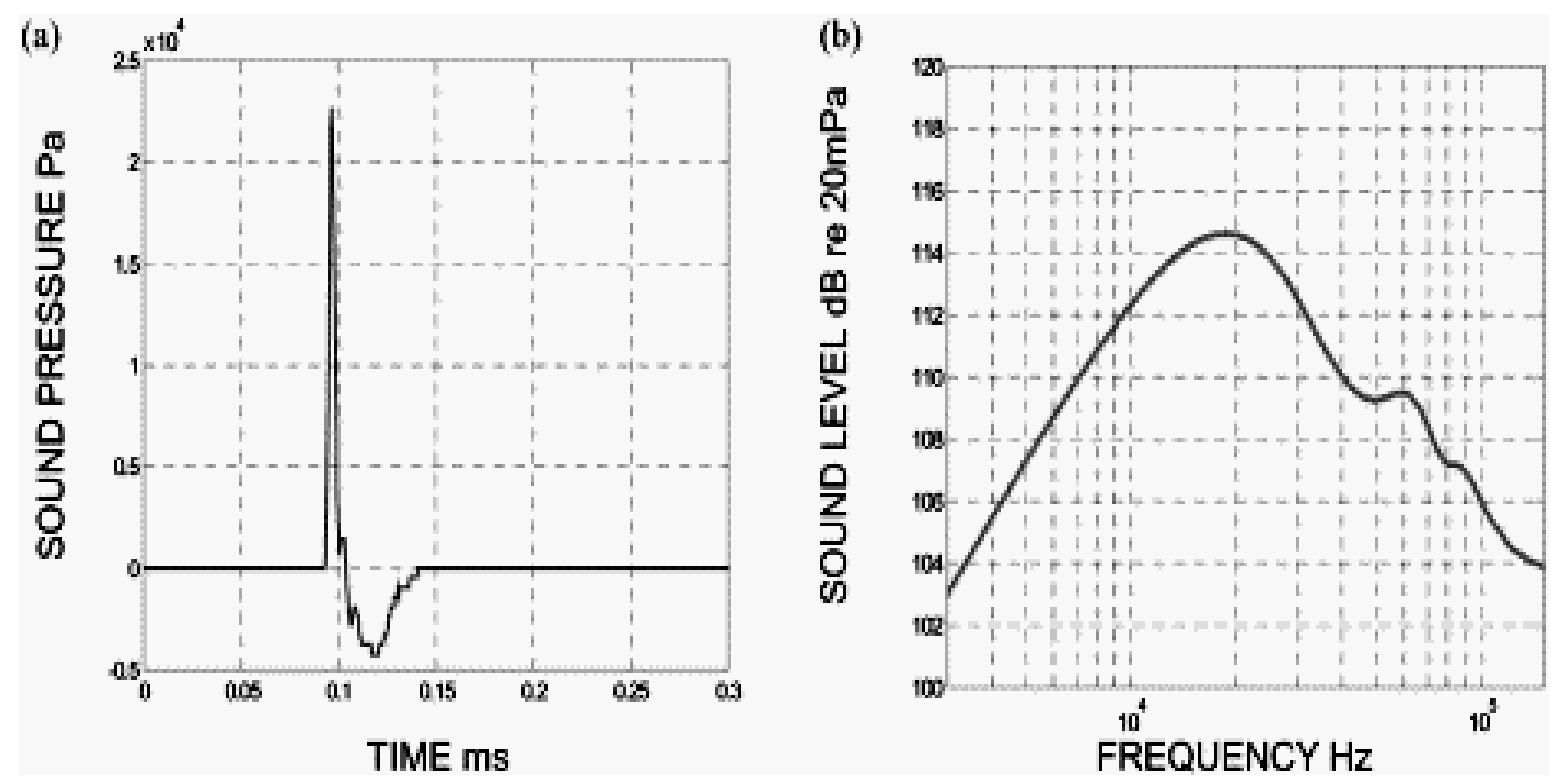

Fig. 4. Measured laser-generated acoustic pulse (a) and the corresponding frequency spectrum (b) at a source-receiver distance of $3 \mathrm{~cm}$ [4]; the peak pressure is $181 \mathrm{~dB}(22683 \mathrm{~Pa})$ and the positive duration is $12 \mu \mathrm{s}$ (reproduced from [4]). 


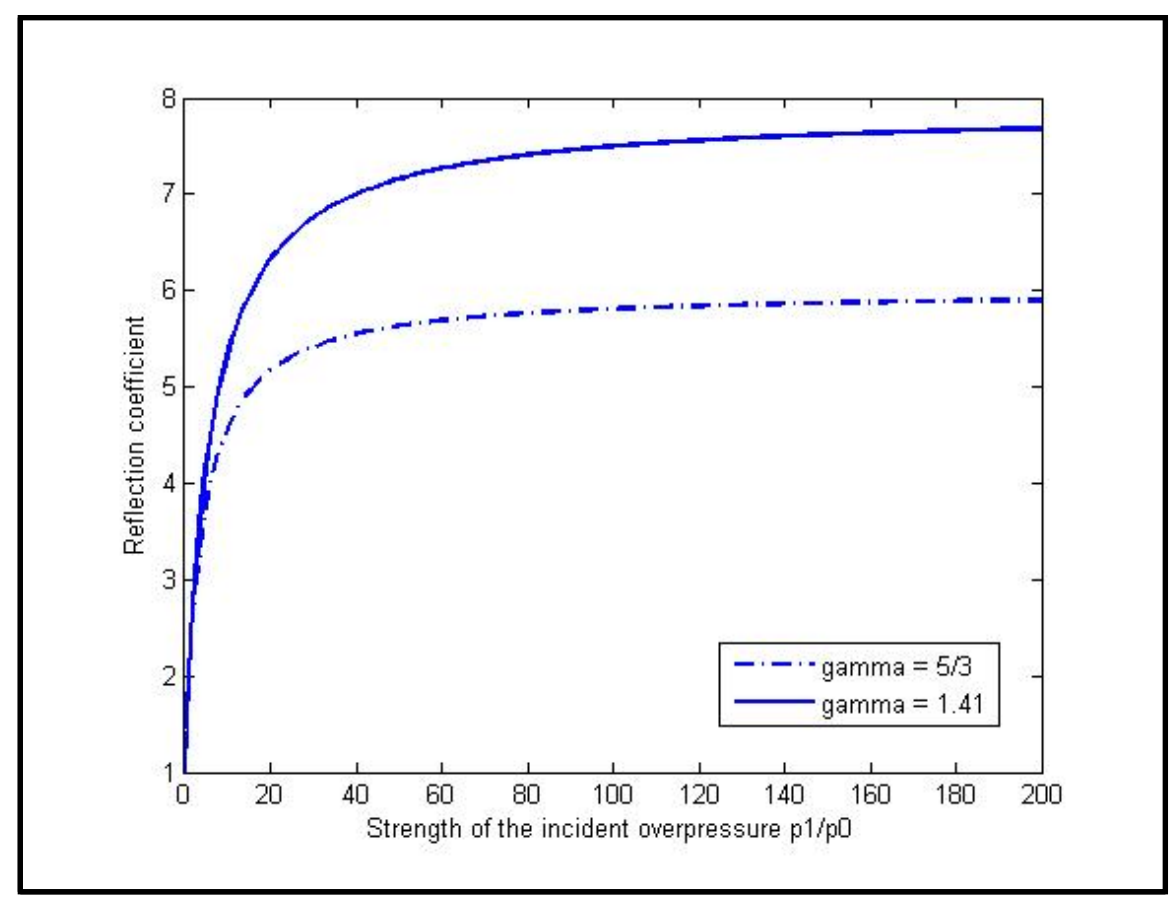

Fig. 5 Reflection coefficient of a shock wave as function of the incident overpressure. 


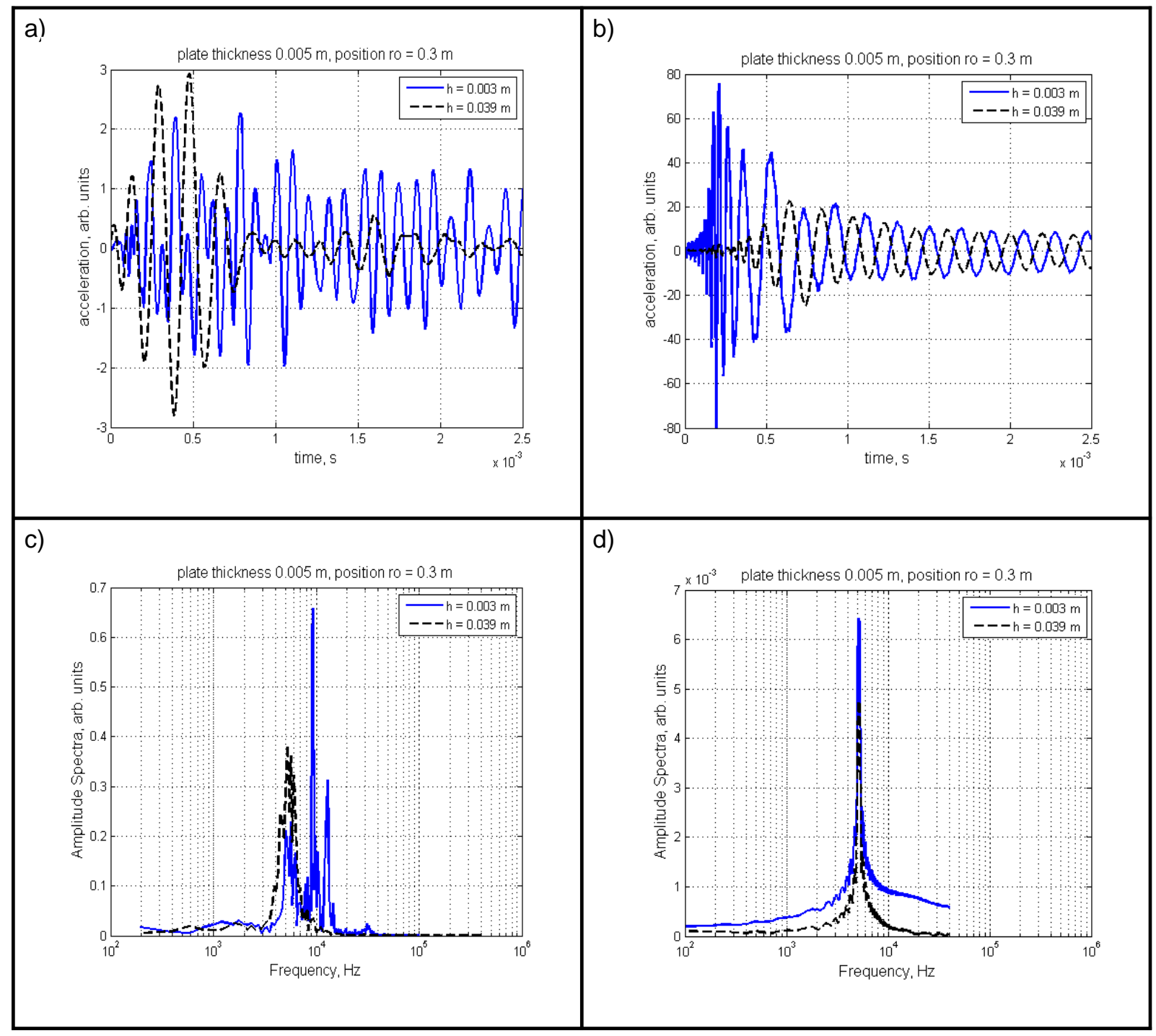

Fig. 6 Time histories ((a), (b)) and amplitude spectra ((c), (d)) of the plate acceleration at distance $0.3 \mathrm{~m}$ away from the epicenter: measured ((a), (c)) and calculated ((b),(d)). 


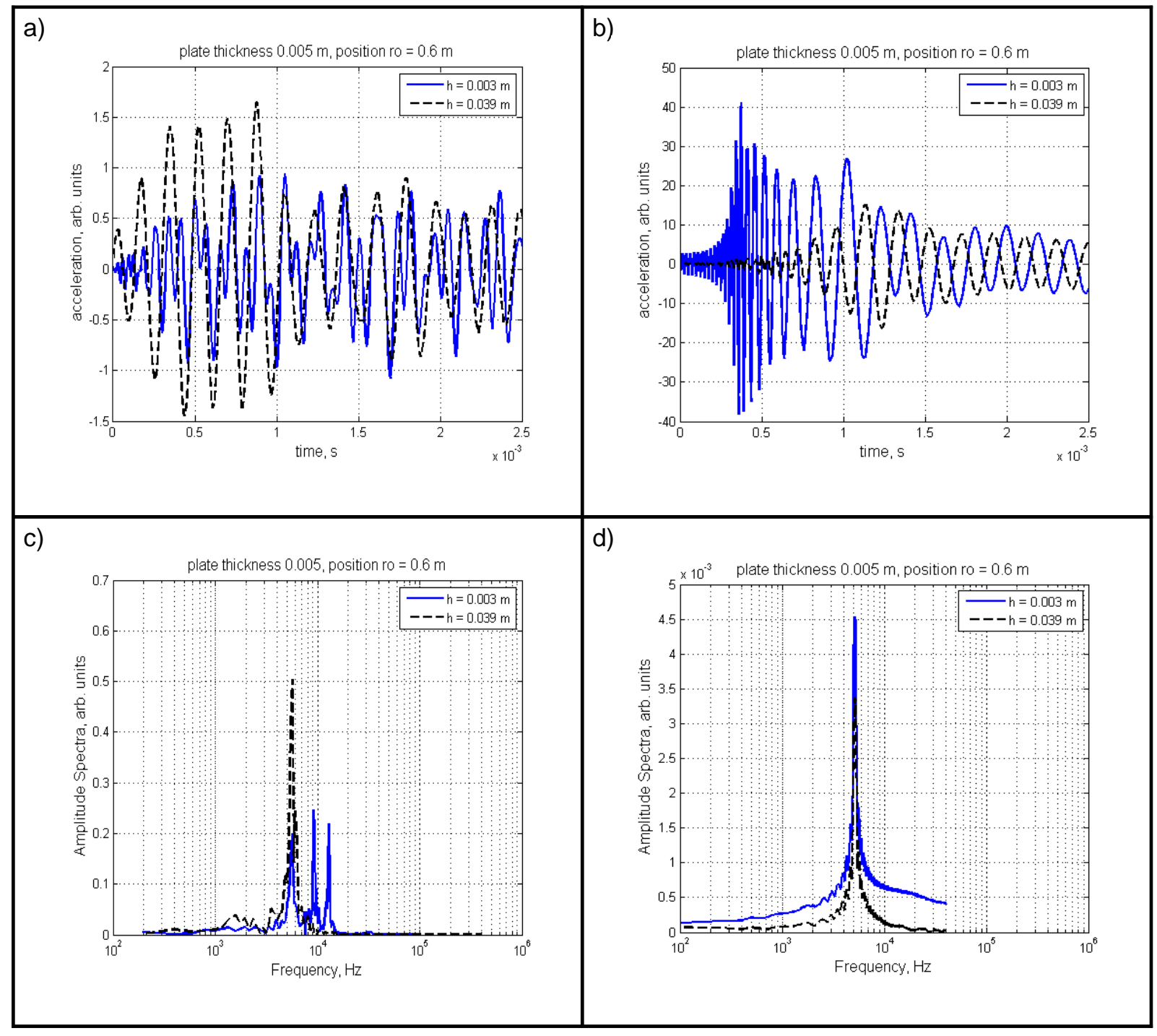

Fig. 7 Time histories ((a), (b)) and amplitude spectra ((c), (d)) of the plate acceleration at distance $0.6 \mathrm{~m}$ away from the epicenter: measured ((a), (c)) and calculated ((b),(d)). 


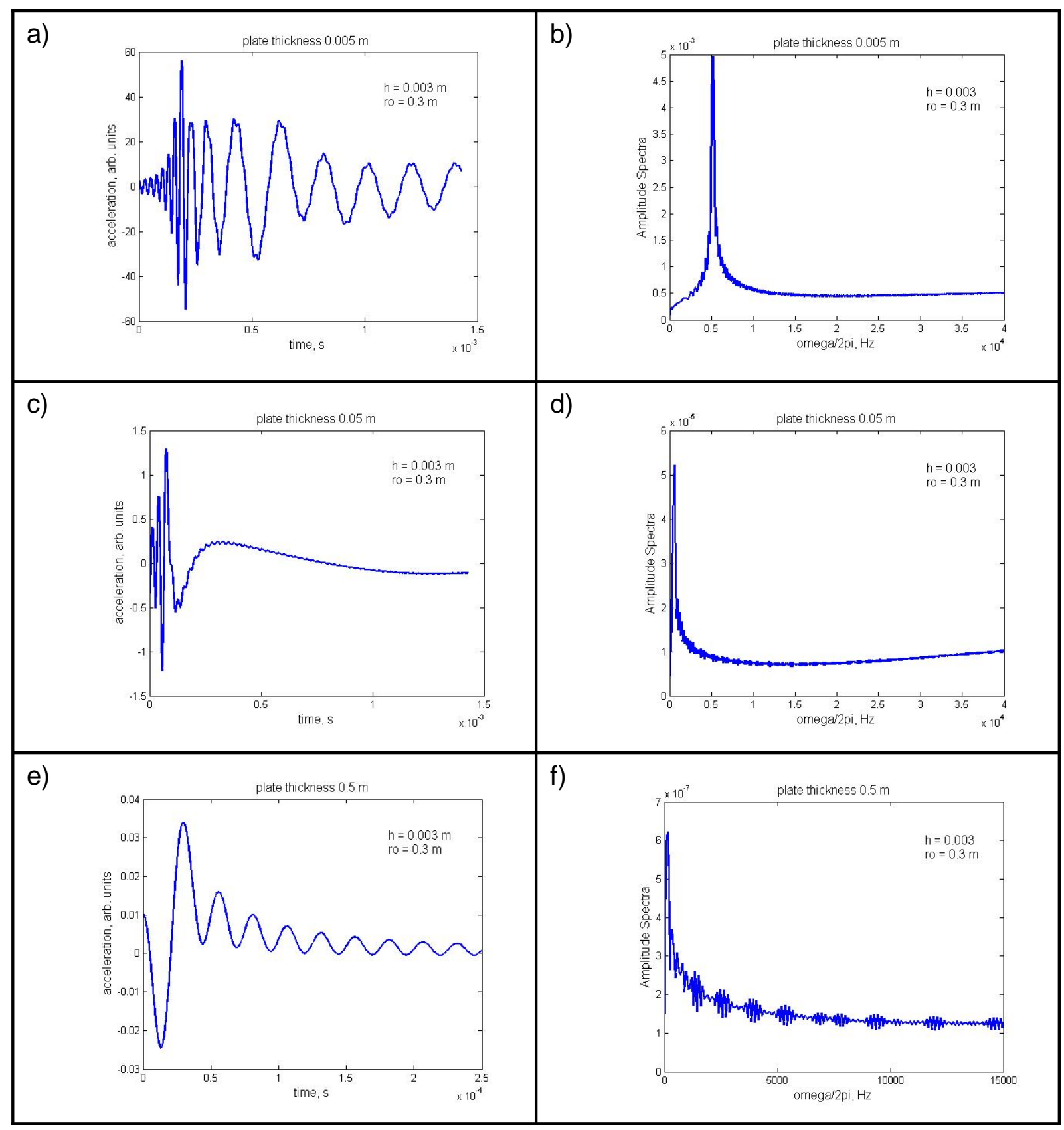

Fig. 8 Time histories (right) and amplitude spectra (left) of the acceleration for different values of plate thickness 


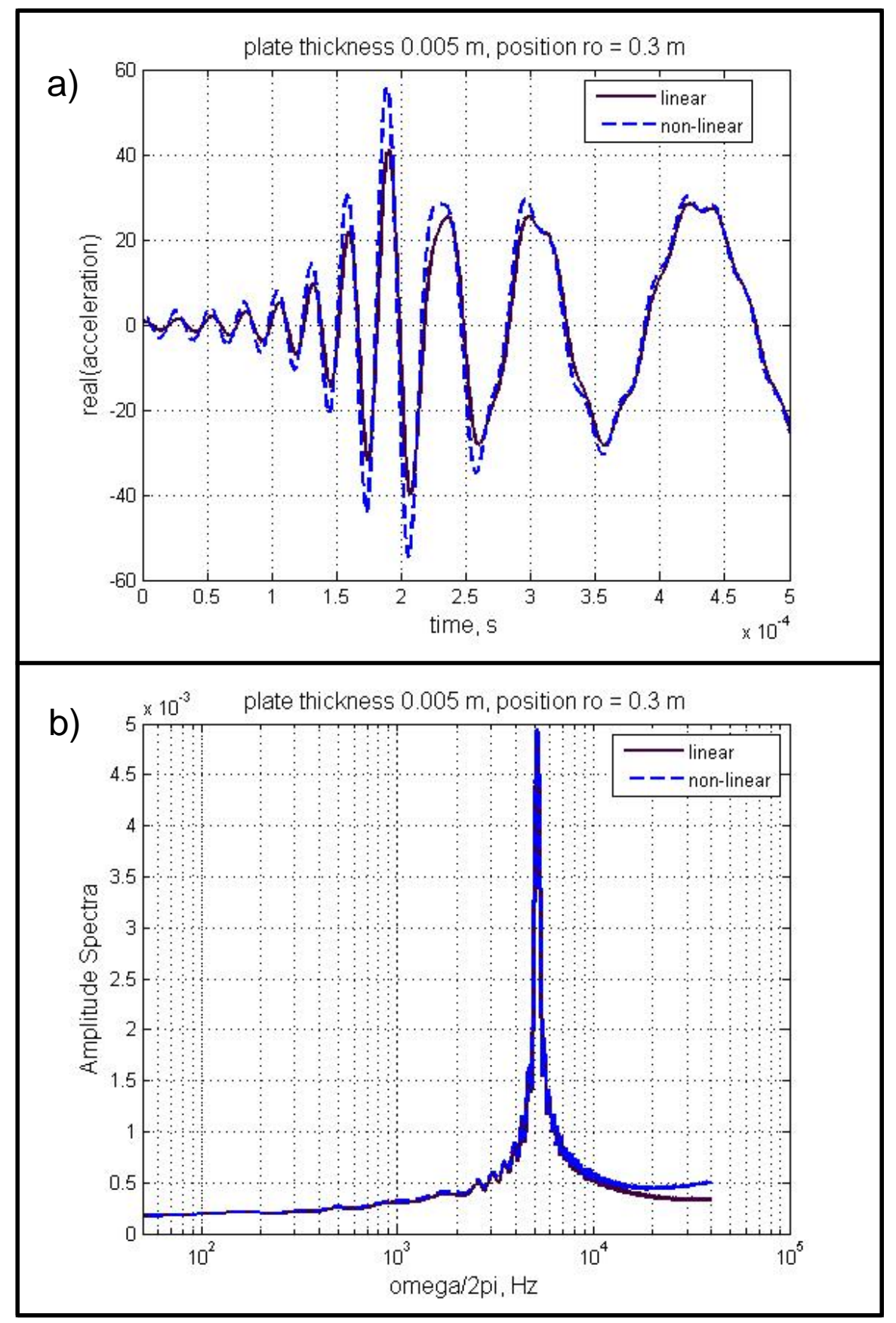

Fig. 9 Effects of linear (solid curves) and non-linear reflection (dashed curves) on time histories (a) and amplitude spectra (b) of plate acceleration. 